\title{
Lactate Levels As A Prognostic Indicator In Heterogeneous ICU Population
}

\author{
Mohamad Ommid ${ }^{1 *}$, Amina Khatoom ${ }^{1}$, Sheikh Ishaq ${ }^{2}$, Reyaz Lone ${ }^{3}$, Saba Ahad ${ }^{1}$, \\ Abraq Asma ${ }^{1}$ and Shahina Parveen ${ }^{1}$ \\ ${ }^{1}$ Department of Anesthesiology Government Medical College Srinagar Jammu and Kashmir India \\ ${ }^{2}$ Department of Biochemistry Government Medical College Srinagar Jammu and Kashmir India \\ ${ }^{3}$ Department of Paediatric Surgery H.M.C Doha Qatar
}

\begin{abstract}
Background: The period course of blood lactate levels could be supportive to evaluate a patient's response to therapy. Serial blood lactate levels in different groups of acutely ill patients.

Methods: A total of 200 patients admitted to ICU with critical illness were randomly selected for the study group, Relevant demographic information, clinical history, clinical and laboratory parameters including diagnosis and length of ICU stay were recorded from each patient. The first sample was taken on admission the other samples was taken on 2nd, 3rd and 4th day of admission. Serial arterial sample lactate measurement was done from the patients to note the initial level, time of maximal level and level at time to normal.

Result: In the study population $27.5 \%$ belonged to the age group of $40-49$ years followed by $24 \%$ in the age group $50-59$ and $20.5 \%$ in 60 69 yrs. $57 \%$ patients were males and $43 \%$ were females. $20.5 \%$ patients expired $34.5 \%$ patients had hyperlactatemia during ICU stay out of which $31.9 \%$ patients expired.

Conclusion: Elevated lactate is encountered in a multitude of clinical presentations. Hyperlactatemia is associated with an in-hospital mortality in a heterogeneous ICU population. Patients with elevated lactate levels predicted in hospital mortality and also normalization of lactate within 6 hours is associated with lower mortality. The observation of a better outcome associated with decreasing blood lactate concentrations was consistent throughout the clinical study. Lactate level is an easily measured laboratory parameter which can provide useful information for the bedside clinician when incorporated into the apt clinical milieu.
\end{abstract}

Keywords: APACHE, Lactate, In-hospital Mortality, Heterogeneous ICU Population.

\section{Introduction}

Many variables measured in critically ill patients have been used to estimate severity of disease, prognosticate morbidity and mortality, evaluate costs of treatment, and finally indicate specific treatment and monitor the adequacy of treatment and its timing. It is unlikely that one measurement can replace all of these, but lactate levels may come close. [1] Septic shock is often associated with macrocirculatory dysfunction causing arterial hypotension, as well as microcirculatory dysfunction, and decreased oxygen and nutrient extraction by peripheral tissues. Lactic acid levels have become a useful marker for tissue hypoperfusion and may also serve as an endpoint for resuscitation in patients with sepsis and septic shock. $[2,3]$. Lactate is produced by most tissues in the human body, with the highest level of production found in muscle. [4] Under normal conditions, lactate is rapidly cleared by the liver with a small amount of additional clearance by the kidneys. [5] In aerobic conditions, pyruvate is produced via glycolysis and then enters the Krebs cycle, largely bypassing the production of lactate.
Under anaerobic conditions, lactate is an end product of glycolysis and feeds into the Cori cycle as a substrate for gluconeogenesis (Figure I)

\section{Aims \& Objective:}

To evaluate the use of lactate measurement in ICU services for earlier detection of occult shock.

To evaluate the use of lactate measurement in ICU services for earlier start of goal-directed therapy in order to, ultimately, influence patient outcome.

To evaluate the use of Lactate levels as a prognostic implication in ICU patients.

To evaluate the use of lactate measurement as a factor for predicting mortality in ICU patients.

To evaluate the cost and cost effectiveness of lactate measurement in ICU patients.

\section{Materials and Methods}

This prospective observational study was conducted in the department of anesthesia and intensive care medicine of 
Government Medical College Srinagar for a period of 1 year after proper ethical consultation and registration with the Institutions' Ethical Clearance Committee. Critically ill patients admitted to ICU of all ages and sex were included in the study. Postoperative patients admitted to ICU for monitoring were excluded.

\section{Methodology}

A total of 200 patients admitted to ICU with critical illness were randomly selected for the study group, Relevant demographic information, clinical history, reason for ICU admission, clinical and laboratory parameters including diagnosis and length of ICU stay were recorded from each patient. The first sample was taken on admission the other samples was taken on $2^{\text {nd }}, 3^{\text {rd }}$ and $4^{\text {th }}$ day of admission. Serial arterial sample lactate measurement was done from the patients to note the initial level, time of maximal level and level at time to normal. We noted the Apache score along with and made a comparative evaluation. Lactate measurement was done from arterial sample using GEM Premier 3000 instrument with reference values 2-3 mmols/dl. Statistical analysis of the data obtained was done on the SPSS software. Data was presented as mean \pm SD or median range. $\mathrm{P}$-value of $<0.05$ was considered statistically significant.

\section{Result}

In the study population maximum number of patients i.e. 55 (27.5\%) belonged to the age group of 40-49 years followed by $48(24 \%)$ in the age group $50-59$ and $41(20.5 \%)$ in $60-69$ yrs. Minimum number of patients i.e. only $7(3.5 \%)$ were above the age of 70 years. Out of which 114 (57\%) patients were males and $86(43 \%)$ were females with a male: female ratio of $1.3: 1$. In our study $41(20.5 \%)$ patients expired and $159(79.5 \%)$ patients survived. In our study $69(34.5 \%)$ patients had hyperlactatemia during ICU stay out of which $22(31.9 \%)$ patients expired and 47(68.1\%) patients survived. $131(65.5 \%)$ patients didn't have hyperlactatemia during ICU stay out of which $19(14.5 \%)$ patients expired and $112(85.5 \%)$ patients survived (Table I)

As far as association of lactate normalization time with outcome in studied patients is concerned out of $28(46.5 \%)$ patients who had normalization time of $\leq 6$ Hours $5(17.9 \%)$ expired and $23(82.1 \%)$ survived. And out of $41(63.5 \%)$ patients who had normalization time of $>6$ Hours $17(41.5 \%)$ patients expired and $24(58.5 \%)$ survived. (Table II)

In Our study mean age of survivors was 48.3 years and mean age of non-survivors was 51.7 years. Mean APACHE Score in survivors and non-survivors was 14.75 and 23.73 respectively. The Length of stay in ICU (Days) in survivors was 2.6 and in non-survivors was 3.4, Lactate level at admission in survivors was $2.31 \mathrm{mmols} / \mathrm{dl}$ and in nonsurvivors was $3.53 \mathrm{mmols} / \mathrm{dl}$ and maximum lactate level in survivors and non-survivors was $2.57 \mathrm{mmols} / \mathrm{dl}$ and 3.95 mmols/dl respectively (Table III).

Table I: Showing association of hyperlactatemia during ICU stay with outcome in studied patients.

\begin{tabular}{|l|c|c|c|c|c|}
\multirow{2}{*}{ Outcome } & \multicolumn{2}{|c|}{ Hyperlactatemia During ICU Stay } & \multicolumn{2}{c|}{ No Hyperlactatemia During ICU Stay } & \%age \\
\cline { 2 - 6 } & No.value & 14.5 & $0.004^{*}$ \\
\hline Expired & 22 & \%age & 19 & 85.5 & 100 \\
\hline Survived & 47 & 68.1 & 112 & 100 \\
\hline
\end{tabular}

Table II: Showing association of lactate normalization time with outcome in studied patients.

\begin{tabular}{|l|c|c|c|c|c|}
\multirow{2}{*}{ Outcome } & \multicolumn{2}{|c|}{$\leq \mathbf{6}$ Hours } & \multicolumn{2}{|c|}{$>\mathbf{6}$ Hours } & P-value \\
\cline { 2 - 5 } & No. & \%age & No. & 41.5 & \\
\hline Expired & 5 & 17.9 & 17 & 58.5 & $0.039 *$ \\
\hline Survived & 23 & 82.1 & 24 & 100 & \\
\hline Total & $\mathbf{2 8}$ & $\mathbf{1 0 0}$ & $\mathbf{4 1}$ & \\
\hline
\end{tabular}

Table III: Comparison on the basis of Age, APACHE Score, LOS in ICU and Lactate levels among Survivors and Non-survivors.

\begin{tabular}{|l|c|c|c|c|c|c|c|}
\multirow{2}{*}{ Characteristic } & \multicolumn{3}{|c|}{ Survivors (n=159) } & \multicolumn{3}{c|}{ Non-survivors (n=41) } \\
\cline { 2 - 8 } & Mean & SD & Range & Mean & SD & Range & P-value \\
\hline Age & 48.3 & 12.01 & $20-72$ & 51.7 & 13.36 & $20-77$ & $0.112^{\#}$ \\
\hline APACHE Score & 14.75 & 3.82 & $9-21$ & 23.73 & 3.93 & $16-30$ & $<0.001^{*}$ \\
\hline LOS ICU (Days) & 2.6 & 1.14 & $1-5$ & 3.4 & 1.91 & $1-8$ & $0.0004^{*}$ \\
\hline Lactate at Adm. (mmol/L) & 2.31 & 0.28 & $1.6-3.0$ & 3.53 & 0.74 & $2.5-5.5$ & $<0.001^{*}$ \\
\hline Max Lactate (mmol/L) & 2.57 & 0.30 & $2.0-3.4$ & 3.95 & 0.79 & $2.9-6.2$ & $<0.001^{*}$ \\
\hline
\end{tabular}




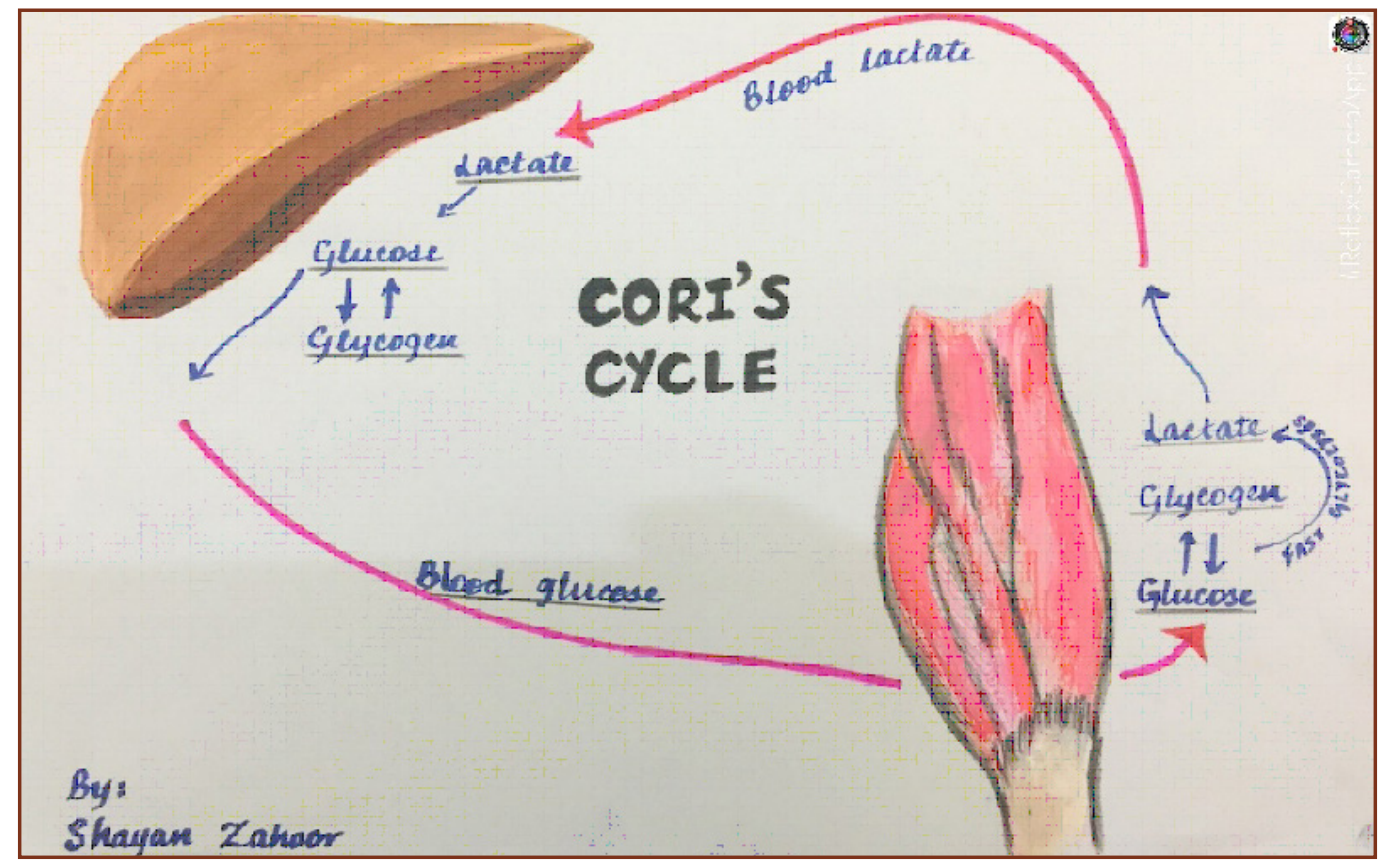

Fig. 1: CORI'S CYCLE.

\section{Discussion}

Risk prediction is an imperative in intensive care settings. The APACHE II score is used as a severity score during the first 24 hours of ICU admission, whereas the SOFA score was developed to evaluate morbidity during ICU stay. Though several clinical and laboratory parameters have been evaluated for the prediction of mortality, easily measurable prognostic biomarker (s) are wanted during ICU stay. In the present study maximum number of patients i.e. 55 belonged to the age group of 40-49 years followed by 48 in the age group 50-59 and 41 in 60-69 yrs. Minimum number of patients i.e. 7 were above 70 years age in which no one survived, the significant independent variable was old age. Old age could predict a worse outcome according to our study which is in concordance with the studies conducted by Arnon Blum et al [6].

In the present study $34.5 \%$ patients had hyperlactatemia during ICU stay out of which $31.9 \%$ patients expired. Our study is in complete agreement with the studies conducted by Khosravani $\mathrm{H}$ et al [7] who concluded Among a cohort of 9107 first admissions with ICU stay of at least one day, both hyperlactatemia at presentation (712/3634 (20\%) vs. $289 / 5473(5 \%) ; \mathrm{P}<0.001)$ and its later development $(101 / 379$ (27\%) vs. $188 / 5094(4 \%) ; \mathrm{P}<0.001)$ were associated with significantly increased case fatality rates as compared with patients without elevated lactate.

In our study mortality rates increased as lactate increased: Lactate level at admission in survivors was $2.31 \mathrm{mmols} /$ $\mathrm{dl}$ and in non-survivors was $3.53 \mathrm{mmols} / \mathrm{dl}$, there was a significant correlation found between the outcome of sepsis and mean lactate level with a $\mathrm{p}$ value $<0.001$. Similar to our study, Shapiro et al [8] with 1278 patients demonstrated that increasing lactate levels were associated with increased mortality in the patients presenting with sepsis. Sustained high lactate levels in ICU patients demonstrated by serial measurements have shown to be predictive of in hospital mortality [9-11] Nichol AD et al in their study concluded that In critically ill patients, relative hyperlactataemia is independently associated with increased hospital mortality. Blood lactate concentrations $>0.75$ mmol.L-1 can be used by clinicians to identify patients at higher risk of death.[12]

As far as association of lactate normalization time with outcome in studied patients is concerned out of $28(46.5 \%)$ patients who had normalization time of $\leq 6$ Hours, 5 (17.9\%) expired and $23(82.1 \%)$ survived. And out of 41 $(63.5 \%)$ patients who had normalization time of $>6$ Hours $17(41.5 \%)$ patients expired and 24(58.5\%) survived.

Nguyen HB et al [9] observed Multivariate logistic regression analysis of statistically significant univariate variables showed lactate clearance to have a significant inverse relationship with mortality $(\mathrm{p}=.04)$. There was an approximately $11 \%$ decrease likelihood of mortality for each $10 \%$ increase in lactate clearance. Haas SA et al [13] made an observation that severe hyperlactatemia $(>10$ $\mathrm{mmol} / \mathrm{L})$ is associated with extremely high ICU mortality especially when there is no marked lactate clearance 
within $12 \mathrm{~h}$. The use of normalized lactate clearance has been associated with improved outcomes in several critical illness settings, including both trauma patients [14,15] and patients with circulatory arrest. [16-18] Bhat SR et al [19] observed that patients who do not clear their lactate in the ED have significantly higher mortality than those with decreasing lactate levels. TC Jansen et al [20] showed that lactate clearance was non-inferior to central venous oxygen saturation (scvO2) as a goal of early resuscitation in patients with severe sepsis or septic shock who presented to the E In this study, two predictive scoring systems have been evaluated in the surgical/medical ICUs. The mean APACHE scores were significantly higher in non-survivors when compared to survivors $(\mathrm{p}<0.001)$. APACHE II score on admission is a standard predictor of mortality in critically ill patients, including septic patients. [21] In our study, the difference of mean APACHE II scores between the survivors and non-survivors was significant and was identified as an independent predictor of mortality in severe sepsis. The findings of our study are in agreement with several studies that higher APACHE II scores were significantly associated with higher mortality rate or poor prognosis. [ 22, 23, 24]

Lactate as a biomarker of disease severity has a number of advantages. First, it can be withdrawn from both peripheral venous or arterial blood, making it possible to obtain lactate levels and to risk-stratify patients early, even in the emergency department. No significant differences exist between central venous, peripheral venous or arterial lactate levels. (25) Second, a number of point-of-care testing (POCT) machines enable speedy, bedside estimation of lactate levels. There seem to be no significant differences between POCT and laboratory lactate results. (26) POCT lactate determination makes sense, especially when goaldirected therapy principles are to be observed. (27) Third, apart from recognition of critically ill patients, lactate tracking can be used as a tool to evaluate the efficacy of treatment not only in sepsis, but in variety of settings. (26) Lastly, the information that raised lactate levels provide, is easily combined into treatment strategies, no additional calculations are needed.

\section{Conclusion}

We concluded that raised lactate is encountered in a multitude of clinical presentations and hyperlactatemia is associated with an in-hospital mortality in a heterogeneous ICU population. Patients with elevated lactate levels predicted in hospital mortality and also normalization of lactate within 6 hours is associated with lower mortality. Lactate level is an easily measured laboratory parameter which can provide useful information for the bedside clinician when incorporated into the apt clinical milieu. Hence blood lactate levels in critically ill patients admitted in ICU seems to be a well-established predictor of prognosis.

\section{Acknowledgements}

The authors would like to acknowledge the figure provided by Miss. Shayan Zahoor (first year MBBS Student), GMC Srinagar

\section{Reference}

1. Jan Bakker, Maarten WN Nijsten and Tim C Jansen; Clinical use of lactate monitoring in critically ill patients. Annals of Intensive Care 2013; 3(12): 1-8.

2. Puskarich MA, Trzeciak S, Shapiro NI, et al. Prognostic value and agreement of achieving lactate clearance or central venous oxygen saturation goals during early sepsis resuscitation. Academic emergency medicine: official journal of the Society for Academic Emergency Medicine. Mar; 2012 19(3):252-258.

3. Nguyen HB, Kuan WS, Batech M, et al. Outcome effectiveness of the severe sepsis resuscitation bundle with addition of lactate clearance as a bundle item: a multinational evaluation. Crit Care. 2011; 15(5): R229.

4. Consoli A, Nurjhan N, Reilly JJ Jr. Bier DM, Gerich JE. Contribution of liver and skeletal muscle to alanine and lactate metabolism in humans. The American journal of physiology. Nov; 1990 259(5 Pt 1):E677-684.

5. Connor H, Woods HF, Ledingham JG, Murray JD. A model of $\mathrm{L}(+)$-lactate metabolism in normal man. Annals of nutrition \& metabolism. 1982; 26(4):254-263.

6. Blum A, Zoubi AA, Kuria S, Blum N. High serum lactate level may predict death within 24 hours. Open Medicine. 2015;10(1):318-322. doi:10.1515/med-2015-0045.

7. Khosravani H, Shahpori R, Stelfox HT, Kirkpatrick AW, Laupland KB. Occurrence and adverse effect on outcome of hyperlactatemia in the critically ill. Critical Care. 2009;13(3):R90. doi:10.1186/cc7918.

8. Shapiro NI1, Howell MD, Talmor D, Nathanson LA, Lisbon A, Wolfe RE, Weiss JW. Serum lactate as a predictor of mortality in emergency department patients with infection. Ann Emerg Med. 2005;45(5):524-528.

9. Nguyen HB, Rivers EP, Knoblich BP, et al. Early lactate clearance is associated with improved outcome in severe sepsis and septic shock. Crit Care Med 2004;32(8):1637-1642

10. Claridge JA, Crabtree TD, Pelltier SJ, et al. Persistent occult hypoperfusion is associated with a significant increase in infection rate and mortality in major trauma patients. $\mathrm{J}$ Trauma 2000;48(1):8-14

11. Jansen TC, van BJ, Mulder PG, et al. Prognostic value of blood lactate levels: does the clinical diagnosis at admission matter? J Trauma 2009;66(2):377-385.

12. Nichol AD, Egi M, Pettila V, et al. Relative hyperlactatemia and hospital mortality in critically ill patients: a retrospective 
multi-centre study. Critical Care. 2010;14(1):R25. doi:10.1186/cc8888.

13. Haas SA, Lange T, Saugel B, Petzoldt M, Fuhrmann V, Metschke M, Kluge S. Severe hyperlactatemia, lactate clearance and mortality in unselected critically ill patients. Intensive Care Med. 2016 Feb;42(2):202-10. doi: 10.1007/ s00134-015-4127-0.

14. Bramson D, Scalea TM, Hitchcock R, et al. Lactate clearance and survival following injury. J Trauma. 1993;35(4):584-588.

15. Blow O, Magliore L, Claridge JA, et al. The golden hour and the silver day: detection and correction of occult hypoperfusion within 24 hours improves outcome from major trauma. J Trauma. 1999;47(5):964-969.

16. Onnino MW, Miller J, Goyal N, et al. Effective lactate clearance is associated with improved outcome in postcardiac arrest patients. Resuscitation. 2007;75(2):229-234.

17. Vincent JL, Dufaye $P$, Berre $J$, et al. Serial lactate determinations during circulatory shock. Crit Care Med. 1983;11(6):449-451.

18. Scott S, Antonaglia V, Guiotto G, et al. Two-hour lactate clearance predicts negative outcome in patients with cardiorespiratory insufficiency. Crit Care Res Pract. 2010;917053

19. Bhat SR, Swenson KE, Francis MW, Wira CR. Lactate Clearance Predicts Survival Among Patients in the Emergency Department with Severe Sepsis. Western Journal of Emergency Medicine. 2015;16(7):1118-1126.

20. Jansen TC, vanbommel J, Schhoonderbeek FJ et al; Early lactate guided therapy in intensive care unit patients: a multicenter, open label, randomized controlled trial. Am J Respir Crit Care Med 2010; 182: 752-761.

21. Knaus WA, Draper EA, Wagner DP, Zimmerman JE. APACHE II: A severity of disease classification system. Crit Care Med 1985;13:818-829.

22. Chen SJ, Chao TF, Chiang MC, Kuo SC, Chen LY, Yin T, et al. Prediction of patient outcome from Acinetobacter baumannii bacteremia with Sequential Organ Failure Assessment (SOFA) and Acute Physiology and Chronic Health Evaluation (APACHE) II scores. Intern Med. 2011;50:871-877.

23. Qiao Q, Lu G, Li M, Shen Y, Xu D. Prediction of outcome in critically ill elderly patients using APACHE II and SOFA scores. J Int Med Res. 2012; 40:1114-1121.

24. Sawicka W, Owczuk R, Wujtewicz MA, Wujtewicz M. The effectiveness of the APACHE II, SAPS II and SOFA prognostic scoring systems in patients with haematological malignancies in the intensive care unit. Anesthesiol Intensive Ther. 2014; 46:166-170.

25. Gallagher EJ, Rodriguez K, Touger M. Agreement between peripheral venous and arterial lactate levels. Ann Emerg Med 1997; 29:479-483

26. Shapiro NI, Fisher C, Donnino M, Cataldo L, Tang A, Trzeciak S, et al. The feasibility and accuracy of point-ofcare lactate measurement in emergency department patients with suspected infection. J Emerg Med 2010; 39:89-94.

27. Goyal M, Pines JM, Drumheller BC, Gaieski DF. Point-ofcare testing at triage decreases time to lactate level in septic patients. J Emerg Med 2010; 38:578-581.

*Corresponding author:

Dr. Mohamad Ommid, Department of Anesthesiology Government Medical College Srinagar Jammu and Kashmir India 190010

Phone: +919419081466

Email: mohamadommid1@gmail.com

Financial or other Competing Interests: None.

Date of Submission : 10.11.2017

Date of Acceptance :05x.12.2017

Date of Publication : 09.12.2017 